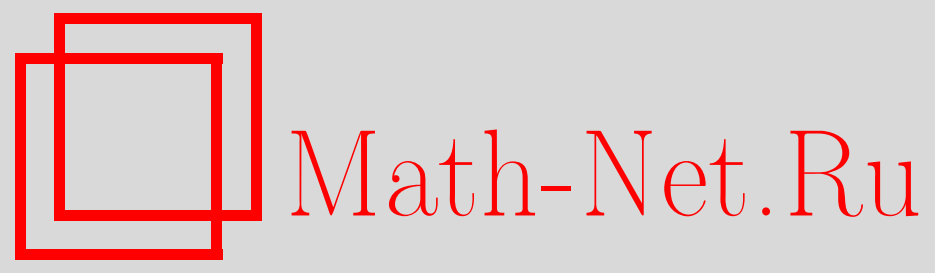

Е. Н. Залесская, О новых классах сопряженных инъекторов конечных групп, Дискрет. матем., 2004, том 16, выпуск $1,105-113$

DOI: https://doi.org/10.4213/dm145

Использование Общероссийского математического портала Math-Net.Ru подразумевает, что вы прочитали и согласны с пользовательским соглашением http://www . mathnet.ru/rus/agreement

Параметры загрузки:

IP: 54.164 .48 .24

26 апреля 2023 г., 16:35:42 


\title{
О новых классах сопряженных инъекторов конечных групп
}

\author{
() 2004 г. Е. Н. Залесская
}

\begin{abstract}
В исследовании задачи существования и сопряженности инъекторов в произвольной конечной группе известен результат Блессеноля-Лауе о том, что в любой конечной группе $G$ существует единственный класс сопряженных квазинильпотентных инъекторов, которые в точности являются $\mathfrak{R}^{*}$-максимальными подгруппами $G$, содержащими обобщенную подгруппу Фиттинга $F^{*}(G)$. В настоящей работе, используя конструкции классов Блессеноля-Лауе и Гашюца, мы расширяем результат Блессеноля-Лауе на случай, когда класс Фиттинга $\mathfrak{F}=\mathfrak{S} \mathfrak{B}$, где $\mathfrak{S}$ - непустой класс Фиттинга и $\mathfrak{B}$ класс Блессеноля-Лауе, тем самым выделяя новый класс сопряженных ґ-инъекторов в классах \& всех конечных групп и $\mathfrak{S}^{\pi}$ всех конечных $\pi$-разрешимых групп соответственно. Более того, мы доказываем, что $\preccurlyeq$-инъекторы группы $G$ - это в точности все

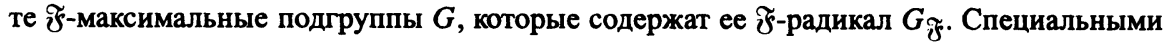
случаями таких инъекторов являются инъекторы для многих известных классов Фиттинга. В частности, такие инъекторы в классе $\subseteq$ всех конечных разрешимых групп были описаны Хартли, Фишером, Францем, Локеттом.
\end{abstract}

\section{1. Введение}

В теории классов Фиттинга конечных разрешимых групा один из основополагающих результатов - обобщение теорем Силова и Холла, которое представляет теорема ГашюцаФишера-Хартли [1].о том, что для любого класса Фиттинга ₹̊ в любой конечной разрешимой группе $G$ существуют $\mathfrak{\Im}$-инъекторы и любые два из них сопряжены в $G$.

Напомним, что класс Фиттинга - класс групп, замкнутый относительно нормальных подгрупп и их произведений, а формация - класс групп, замкнутый относительно гомоморфных образов и конечных подпрямых произведений. Если класс групп одновременно является классом Фиттинга и формацией, то его называют формацией Фиттинга. Заметим, что если $\mathfrak{F}$ - класс Фиттинга, то подгруппа $V$ группы $G$ называется ее $\preccurlyeq$-инъектором [2], если $V \cap N$ является ґ-максимальной подгруппой в $N$ для любой субнормальной подгрупшы $N$ групшы $G$.

Расширенне указанного выше результата на случай произвольных конечных групп в общем случае невозможно (см., например, стр. 295 в [2]). Однако в этом направлении известны результаты Л. А. Шеметкова [3, 4], В. Г. Сементовского [5], которые выделяют классы сопряженных инъекторов в случае, когда группа $G$ частично разрешима.

Впервые классы сопряженных инъекторов в произвольной конечной группе были найдены Блессенолем и Лауе [6]. При этом, ярким результатом в этом направлении является теорема о том, что любая конечная группа $G$ имеет единственный класс сопряженных 
квазинильпотентных инъекторов $\left(\mathfrak{N}^{*}\right.$-инъекторов), которые в точности являются $\mathfrak{N}^{*}$-максимальными подгруппами $G$, содержащими обобщенную подгруппу Фиттинга $F^{*}(G)$.

Напомним, что класс всех квазинильпотентных групп $\mathfrak{R}^{*}$ - это класс групा $\left(G \mid G=F(G) L(G)=F^{*}(G)\right)$, где $F(G)$ - подгруппа Фиттинга, $L(G)$ - полупростой радикал групшы $G$ (см. [7]).

В настоящей работе, используя классы Блессеноля-Лауе [6], а также конструкции классов Фиттинга, предложенные Гашюцом (см. IX.2 в [2]), мы находим новые классы сопряженных инъекторов в классе \& всех конечных групп и классе $\mathfrak{\varsigma}^{\pi}$ всех конечных $\pi$-разрешимых групп. Заметим, что специальными случаями таких инъекторов являются инъекторы для многих известных классов Фиттинга. В частности, такие инъекторы в классе $\sqrt{ }$ всех конечных разрешимых групп были описаны Хартли [8], Фишером [9], Францем [10], Локеттом [11].

Для описания инъекторов мы будем использовать понятие произведения классов Фиттинга. Произведением классов Фиттинга $\mathfrak{X}$ и $\mathfrak{V}$ называется класс всех тех групп $G$, для которых $G / G_{\mathfrak{X}} \in \mathfrak{Y}$.

Рассматриваются только конечные групाы. В определениях и обозначениях мы следуем [2].

\section{2. Предварительные сведения}

\section{1. $B L$-классы}

Вначале напомним процедуру построения классов Фиттинга, которая была предложена Блессенолем и Лауе [6].

Пусть $\mathfrak{Q}$ - подкласс класса $\mathfrak{I}$ всех простых групп, $\boldsymbol{J}$ - произвольная простая группа и $K(J)$ - подгруппа Aut $(J)$, которая удовлетворяет следующим требованиям:

(1) если $J$ и $J_{1}-$ изоморфные простые группы и $\psi-$ изоморфизм $\operatorname{Aut}(J)$ на $\operatorname{Aut}\left(J_{1}\right)$, то $K(J)^{\psi}=K\left(J_{1}\right)$;

(2) если $J$ - циклическая группа, то $K(J)=1$;

(3) для каждой простой группы $J$ справедливо включение $\operatorname{Inn}(J) \leqslant K(J)$.

Тогда ввиду (1) $K(J) \triangleleft \operatorname{Aut}(J)$ для любой простой групшы $J$.

Если $L / M$ - главный фактор групшы $G$ и $L / M$ является прямым произведением изоморфных друг другу простых групп $J_{1}, \ldots, J_{r}$, то $C_{G}^{K}(L / M)$ - нормальная подгруппа $G$, которая определяется следующим образом: $g \in C_{G}^{K}(L / M)$ тогда и только тогда, когда для $g \in G$ справедливо равенство $J_{i}^{g}=J_{i}$ и $g$ индуцирует на $J_{i}$ автоморфизм из $K\left(J_{i}\right)$ для $1 \leqslant i \leqslant r$.

Главный фактор $L / M$ группы $G$ называют $K$-центральным в $G$, если $C_{G}^{K}(L / M)=G$.

Пусть подгрупшы $T$ и $S$ нормальны в $G$ и $T \subseteq S$, тогда под главным фактором $G$ между $T$ и $S$ понимают какой-нибудь $G$-главный фактор $G$-группы $S / T$. Если он является минимальной нормальной подгруппой группы $G / T$, то такой фактор называется минимальным главным фактором $G$ между $S$ и $T$. Главный фактор $L / M$ групшы $G$ называют $\mathfrak{L}$-фактором, если он является прямым произведением простых групп, принадлежащих $\mathfrak{L}$.

Пусть $\mathfrak{X}$ и $\mathfrak{V}$ - классы Фиттинга, причем $\mathfrak{V} \subseteq \mathfrak{X}$. Блессенолем и Лауе [6] были введены два класса $\mathfrak{B}=\mathfrak{B}_{\mathfrak{L}}^{K}(\mathfrak{X}, \mathfrak{V})$ и $\mathfrak{S}=\mathfrak{\mho}_{\mathfrak{L}}^{\mathfrak{\Re}}(\mathfrak{X}, \mathfrak{V})$, которые определяются следующим образом: 
$G \in \mathfrak{B}_{\mathfrak{R}}^{\Re}(\mathfrak{X}, \mathfrak{Y})$ тогда и только тогда, когда каждый главный $\mathfrak{L}$-фактор $G$ между $G_{\mathfrak{X}}$ и $G_{\mathfrak{⿰}}$ $\widetilde{K}$-централен в $G$;

$G \in \mathfrak{\Im}_{\mathfrak{L}}^{K}(\mathfrak{X}, \mathfrak{V})$ тогда и только тогда когда каждый минимальный главный $\mathfrak{L}$-фактор $G$ между $G_{\mathfrak{X}}$ и $G_{\mathfrak{⿰}} K$-централен в $G$.

Классы $\mathfrak{B}$ и $\mathfrak{5}$ назовем классами Блессеноля-Лауе или коротко $B L$-классами. Как установлено [6] (см. теорему 1.2), $B L$-классы являются классами Фиттинга.

\section{2. $B L$-классы и конструкции Гашюца}

Композищионной функщией Хартли или композищионной $\boldsymbol{H}$-функцией назовем функцию $f: \mathfrak{I} \rightarrow$ \{классы Фиттинга $\},$ принимающую одинаковые значения на изоморфных группах. Если функция $f: \mathfrak{I} \rightarrow$ \{формации\} принимает одинаковые значения на изоморфных группах, то $f$ называют (см. [12]) композиционным экраном или композиционным спутником.

Пусть $\mathfrak{\lessgtr}$ - такая формация Фиттинга, что $D_{0}(J) \subseteq \mathfrak{F} \subseteq D_{0} S_{n}(\operatorname{Aut}(J))$.

Определим композищионную $H$-функцию $f$ следующим образом:

$$
f(J)= \begin{cases}(1), & J \in \mathfrak{R} \cap \mathfrak{A}, \\ \mathfrak{F}, & J \in \mathfrak{L} \backslash \mathfrak{A}, \\ \mathfrak{F}, & J \in \mathfrak{S} \backslash \mathfrak{R}\end{cases}
$$

Напомним, что если $f$ - композиционный спутник и $L / M$ - нормальная секция группы $G$, то $L / M$ называют $f$-гиперцентральной в $G$, если каждый $G$-главный $\mathfrak{Q}$-фактор $R / S$ группы $L / M$ является $f$-центральным в $G$, то есть $G / C_{G}(R / S) \in f(J)$ для всех $J \in \mathfrak{L}$. Следуя Гашюцу (см. IX.2 в [2]), пусть $\mathfrak{X}$ и $\mathfrak{Y}-$ классы Фиттинга, причем $\mathfrak{X} \subseteq \mathfrak{V}$ и

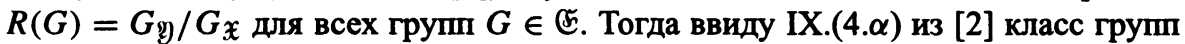

$$
H R(f, R)=\{G \in \mathbb{E} \mid R(G) f \text {-гиперцентральна в } G\}
$$

совпадает с $B L$-классом $\mathfrak{B}$ и является классом Фиттинга.

Если же $\psi$ - такая композищионная $H$-функция, что

$$
\psi(J)= \begin{cases}(1), & J \in \mathfrak{L} \cap \mathfrak{A}, \\ D_{0}(J), & J \in \mathfrak{L} \backslash \mathfrak{A}, \\ \mathbb{E}, & J \in \mathfrak{I} \backslash \mathfrak{L},\end{cases}
$$

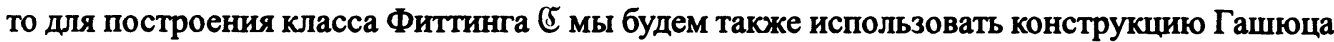
(см. IX.2 в [2]).

При этом напомним, что цоколем группы $G$ называется произведение всех минимальных нормальных подгрупп группы $G$.

Пусть $S(G)=\left(G_{\mathfrak{y}} / G_{\mathfrak{X}}\right) \cap \operatorname{Soc}\left(G / G_{\mathfrak{X}}\right)$ для всех групп $G \in$ \&. Тогда класс групп

$$
H S(\psi, S)=\{G \in \text { ⿷匚 } \mid S(G)\} \psi \text {-гиперцентральна в } G\}
$$

ввиду IX.2.8 из [2] является классом Фиттинга и кроме того (см. стр. 629 в [2]) совпадает с $B L$-классом $巨$.

В дальнейшем мы будем использовать свойства $B L$-классов $\mathfrak{B}$ и $\Subset$ и свойства инъекторов, которые приведем в виде следующих двух лемм.

Пусть $\pi=\operatorname{Char}(\mathfrak{L})$ и $S^{\pi}$ - класс всех $\pi$-разрешимых групп. Тогда с учетом леммы IX.4.2 из [2] и теорем 4.1 и 4.2 из [3] справедливо следующее утверждение. 
Лемма 1. Для классов $\mathfrak{B}$ и 5 справедливы следующие утверждения:

(1) в любой группе $G \in$ \& существует единственный класс сопряжсенных $\mathfrak{B - и н ъ е к т о - ~}$ ров, которые в точности являются $\mathfrak{B}$-максимальными подгруппами $G$, содержачими $\mathfrak{B - р а д и к а л ~ г р у п п ы ~} G$;

(2) в любой группе $G \in \mathfrak{S}^{\pi}$ существует единственный класс сопряженных $\mathfrak{}$-инъекто-

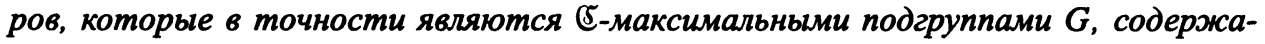
ииими ङ-радикал группы $G$.

Лемма 2 ([2]). Пусть $G$ - произвольная группа и $\mathfrak{F}$ - непустой класс Фиттинга. Тогда справедливы следуючие утверждения:

(1) если $V-\mathfrak{\Im}$-инъектор $G u K \triangleleft G$, то $V \cap K-\mathfrak{s}$-инъектор группы $K$;

(2) если $V-\mathfrak{\Im}$-инъектор $G u \alpha: G \rightarrow G \alpha$ изоморфизм, то $V \alpha$ является $\mathfrak{\Im}$-инъектором групnbl $G \alpha$;

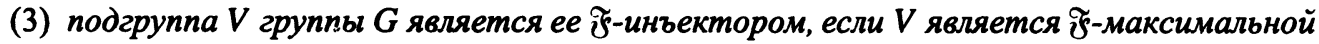

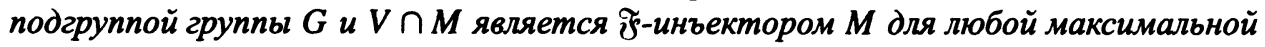
нормальной подгруппы $M$ группы $G$.

\section{3. Инъекторы произведений классов Фиттинга}

Если $\mathfrak{f}$ - непустой класс Фиттинга, то группу $G$ называют ₹̧-скованной [13], если $C_{G}\left(G_{\mathfrak{⿰}}\right) \leqslant G_{\Im}$.

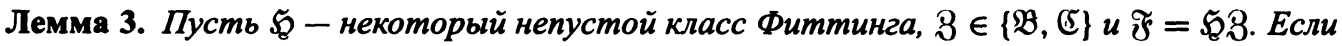
$G$ - такая группа, что группа $G / G_{\mathfrak{G}}$ 3-скована, $u V$ - подгруппа группы $G$, содержсащая еe ₹ో-радикал $G_{\mathfrak{⿰}}$, то справедливы следуючие утверждения:

(1) $V_{\mathfrak{B}}=G_{\mathfrak{g}}$;

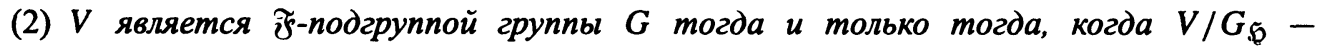
3-noдгрупnа групnы $G / G_{\mathfrak{\$}}$;

(3) подгруппа $V$ F-максимальна в $G$ тогда и только тогда, когда подгруппа $V / G_{\mathfrak{b}}$ 3-максимальна в $G / G_{\mathfrak{5}}$.

Доказательство. 1. По условию $G_{\mathfrak{F}} \triangleleft V$ и $\mathfrak{S} \subseteq \mathfrak{F}$. Следовательно,

$$
\left[V_{\mathfrak{W}}, G_{\mathfrak{F}}\right] \subseteq V_{\mathfrak{W}} \cap G_{\mathfrak{F}}=\left(G_{\mathfrak{F}}\right)_{\mathfrak{W}}=G_{\mathfrak{W}}
$$

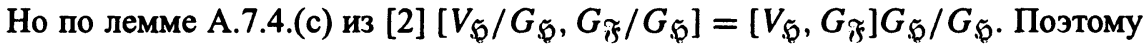

$$
\left[V_{\mathfrak{S}}, G_{\mathfrak{F}}\right] G_{\mathfrak{W}} / G_{\mathfrak{\mathfrak { W }}} \subseteq G_{\mathfrak{W}} / G_{\mathfrak{W}}
$$

Следовательно, подгрупшы $V_{\mathfrak{W}} / G_{\mathfrak{W}}$ и $G_{\mathfrak{F}} / G_{\mathfrak{W}}$ поэлементно перестановочны. Значит, $V_{\mathfrak{W}} / G_{\mathfrak{W}} \subseteq C_{G / G_{\mathfrak{q}}}\left(G_{\mathfrak{F}} / G_{\mathfrak{W}}\right)$. Но $C_{G / G_{\mathfrak{W}}}\left(\left(G / G_{\mathfrak{W}}\right)_{\mathfrak{Z}}\right) \subseteq\left(G / G_{\mathfrak{h}}\right)_{\mathfrak{3}}$. Ввиду леммы IX.1.12(b) из [2] $\left(G / G_{\mathfrak{W}}\right)_{\mathfrak{3}}=G_{\mathfrak{b} 3} / G_{\mathfrak{W}}=G_{\mathfrak{F}} / G_{\mathfrak{\mathfrak { W }}}$, и поэтому $C_{G / G_{\mathfrak{Q}}}\left(G_{\mathfrak{F}} / G_{\mathfrak{W}}\right) \subseteq G_{\mathfrak{F}} / G_{\mathfrak{\mathfrak { b }}}$.

Таким образом, $V_{\mathfrak{g}} / G_{\mathfrak{g}} \subseteq G_{\mathfrak{F}} / G_{\mathfrak{5}}$ и $V_{\mathfrak{g}} \subseteq G_{\mathfrak{F}}$. Следовательно,

$$
G_{\mathfrak{Q}}=\left(G_{\mathfrak{T}}\right)_{\mathfrak{W}}=V_{\mathfrak{W}} \cap G_{\mathfrak{F}}=V_{\mathfrak{D}}
$$


2. Так как $G_{\mathfrak{F}} \subseteq V$, то по утверждению 1 леммы $3 V_{\mathfrak{g}}=G_{\mathfrak{g}}$. Следовательно, $V$ является $\mathfrak{\Im}$-подгруппой в точности тогда, когда $V / G_{\mathfrak{g}}$ является 3 -группой.

3. Пусть $V-\mathfrak{F}$-максимальная подгруппа групшы $G$. Предположим, что $V / G_{\mathfrak{G}} \subset L / G_{\mathfrak{G}} \in 3$. Ввиду того, что $G_{\mathfrak{F}} \subset L$, по утверждению 1 леммы $3 L_{\mathfrak{b}}=G_{\mathfrak{W}}$. Следовательно, $L / G_{\mathfrak{S}}=L / L_{\mathfrak{S}} \in \mathbb{Z}$ и $L \in \mathfrak{F}$. Получили противоречие с тем, что подгрупша $V$ является $\mathfrak{F}$-максимальной в $G$. Значит, $V=L$ и подгруппа $V / G_{\mathfrak{G}}$ является 8-максимальной в $G / G_{\mathfrak{S}}$.

Пусть теперь подгруппа $V / G_{\mathfrak{S}}$ является 3 -максимальной в $G / G_{\mathfrak{g}}$. Предположим, что $V \subset L \in \mathfrak{F}$. Тогда из того, что $G_{\mathfrak{F}} \subset L$, по утверждению 1 леммы 3 следует, что $L_{\mathfrak{G}}=G_{\mathfrak{b}}$. Так как $L \in \mathfrak{F}$, то $L / L_{\mathfrak{W}} \in 3$. Значит, $V / G_{\mathfrak{W}} \subset L / G_{\mathfrak{W}}=L / L_{\mathfrak{G}} \in 3$. Это противоречит тому, что $V / G_{\mathfrak{b}}$ является 3 -максимальной в $G / G_{\mathfrak{5}}$. Следовательно, $V=L$ и подгруппа $V$ является $\mathfrak{F}$-максимальной в $G$. Лемма доказана.

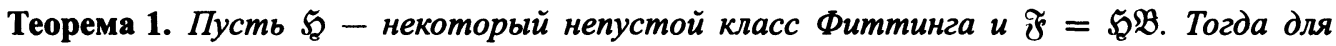
любой группы $G$ такой, что $G / G_{\mathfrak{g}}$ является $\mathfrak{B}$-скованной, и ее подгруппы $V$ справедливы следующие утверждения:

(1) $V$ является $\mathfrak{s}$-инъектором группы $G$ тогда и только тогда, когда $V / G_{\mathfrak{b}}$ является B-инъектором группы $G / G_{\mathfrak{g}}$;

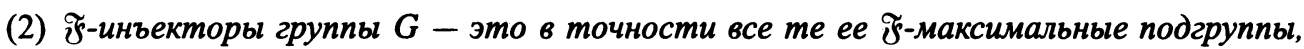
которые содержат ₹-радикал $G_{\mathfrak{F}}$;

(3) в любой группе $G$ существуют $\mathfrak{\$}$-инъекторы и любые два из них сопряжены в $G$.

Доказательство. Докажем первое утверждение. Пусть $V-\mathfrak{\wp}$-инъектор группы $G$. Тогда $G_{\mathfrak{\jmath}} \subseteq V$ и $V$ является $\mathfrak{\lessgtr}$-максимальной в $G$. Следовательно, по утверждению 3 леммы 3 подгруппа $V / G_{\mathfrak{5}}$ является $\mathfrak{B}$-максимальной в $G / G_{\mathfrak{b}}$. Ввиду леммы IX.1.12(b) из [2] $\left(G / G_{\mathfrak{W}}\right)_{\mathfrak{B}}=G_{\mathfrak{\zeta}} / G_{\mathfrak{W}}$. Поэтому из того, что $G_{\mathfrak{F}} \subseteq V$, следует, что $\left(G / G_{\mathfrak{W}}\right)_{\mathfrak{B}} \subseteq V / G_{\mathfrak{W}}$. Отсюда по утверждению 1 леммы 1 получаем, что $V / G_{\mathfrak{b}}$ есть $\mathfrak{B}$-инъектор группы $G / G_{\mathfrak{b}}$.

Обратное утверждение докажем индукцией по порядку группы $G$. Пусть $G$ - контрпример минимального порядка. Согласно утверждению 1 леммы 1 в группе $G / G_{\mathfrak{g}}$ существует $\mathfrak{B}$-инъектор $V / G_{\mathfrak{b}}$. Пусть $M$ - любая максимальная нормальная подгруппа группы $G$. Ввиду леммы IX.1.1.(а) из [2] $M_{\mathfrak{W}}=G_{\mathfrak{W}} \cap M$. Рассмотрим два возможных случая.

1. Группа $G_{\mathfrak{W}} \subseteq M$. Тогда $G_{\mathfrak{W}}=M_{\mathfrak{W}}$. Так как $V / G_{\mathfrak{Q}}-\mathfrak{B}$-инъектор групшы $G / G_{\mathfrak{W}}$, по утверждению 1 леммы 2 подгруппа $V \cap M / G_{\mathfrak{g}}$ является $\mathfrak{B}$-инъектором групшы $M / G_{\mathfrak{g}}$. Но в данном случае $M_{\mathfrak{W}}=G_{\mathfrak{W}}$, и поэтому $V \cap M / M_{\mathfrak{W}}-\mathfrak{B}$-инъектор групшы $M / M_{\mathfrak{D}}$. Следовательно, по индукции подгруппа $V \cap M-\mathfrak{s}$-инъектор группы $M$. Так как $V / G_{\mathfrak{S}} \in \mathfrak{B}$, про утверждению 2 леммы $3 V \in \mathfrak{F}$. Докажем, что $V-\mathfrak{\$}$-максимальная подгруппа группы $G$. Действительно, ввиду утверждения 1 леммы $1 V / G_{\mathfrak{D}}-$ B-максимальная подгруппа группы $G / G_{\mathfrak{g}}$, содержащая подгруппу $\left(G / G_{\mathfrak{p}}\right)_{\mathfrak{B}}$. Так как по лемме IX.1.12 (b) из [2] $\left(G / G_{\mathfrak{b}}\right)_{\mathfrak{B}}=G_{\mathfrak{F}} / G_{\mathfrak{\mathfrak { g }}}$, то $G_{\mathfrak{F}} \subseteq V$. Следовательно, по утверждению 3 леммы $3 V$ является i̧-максимальной в $G$.

Итак, в данном случае $V-\mathfrak{\xi}$-инъектор группы $G$ по утверждению 3 леммы 2 . Полученное противоречие исключает случай 1 . Остается принять случай 2.

2. Группа $G_{\mathfrak{b}} \nsubseteq M$. В этом случае, ввиду максимальности $M, G=G_{\mathfrak{p}} M$. Так как

$$
G / G_{\mathfrak{q}} \simeq M / G_{\mathfrak{j}} \cap M=M / M_{\mathfrak{j}}
$$


по утверждению 2 леммы 2 подгруппа $V \cap M / M_{\mathfrak{G}}$ является $\mathfrak{B}$-инъектором групшы $M / M_{\mathfrak{S}}$. Следовательно, по индукции $V \cap M-\mathfrak{F}$-инъектор групшы $M$. По утверждению 2 леммы 3 $V \in \mathfrak{f}$, и аналогично, как и в случае $1, V$ является $\mathfrak{\wp}$-максимальной в $G$.

Значит, ввиду произвольности выбора максимальной нормальной подгрупшы $M$ группы $G$ из утверждения 3 леммы 2 следует, что $V-\mathfrak{\Im}$-инъектор групшы $G$. Полученное противоречие завершает доказательство первого утверждения теоремы.

Если подгруппа $V$ является $\preccurlyeq$-инъектором группы $G$, то по определению §-инъектора, очевидно, что $G_{\text {§ }} \subseteq V$ и $V$ является ई-максимальной в $G$.

Докажем обратное утверждение. Пусть $V$ - любая $\mathfrak{乛}$-максимальная подгруппа групшы $G$, содержащая ее $\mathfrak{F}$-радикал $G_{\mathfrak{F}}$. Покажем, что $V-\mathfrak{F}$-инъектор групшы $G$. Так как $G_{\mathfrak{F}} \subseteq V$ и $V \in \mathfrak{F}$, по утверждению 2 леммы $3 V / G_{\mathfrak{b}} \in \mathfrak{B}$. Тогда, применяя утверждение 3 этой леммы, заключаем, что подгруппа $V / G_{\mathfrak{g}}$ является $\mathfrak{B}$-максимальной в $G / G_{\mathfrak{g}}$. Но из того, что $G_{\mathfrak{F}} \subseteq V$, следует, что

$$
\left(G / G_{\mathfrak{Q}}\right)_{\mathfrak{B}}=G_{\mathfrak{F}} / G_{\mathfrak{S}} \subseteq V / G_{\mathfrak{W}}
$$

Следовательно, по утверждению 1 леммы $1 V / G_{\mathfrak{Q}}$ является $\mathfrak{B}$-инъектором групшы $G / G_{\mathfrak{W}}$. Но тогда из первого утверждения теоремы вытекает, что подгруппа $V$ является $\mathfrak{5}$-инъектором групшы $G$. Второе утверждение теоремы доказано.

Ввиду утверждения 1 леммы 1 в любой групте $G$ существуют $\mathfrak{B}$-инъекторы и любые два из них сопряжены в $G$. Следовательно, из первого утверждения теоремы вытекает, что в любой группе $G$ существуют $\mathfrak{5}$-инъекторы и любые два из них сопряжены в $G$. Теорема доказана.

Теорема 2. Пусть $\mathfrak{g}$ - некоторый непустой класс Фиттинга $и \mathfrak{F}=\mathfrak{S} \Subset$. Тогда для любой группы $G \in \mathbb{S}^{\pi}$ такой, что группа $G / G_{\mathfrak{\$}}$ является $V$ справедливы следующие утверждения:

(1) $V$ является $\mathfrak{\Im}$-инъектором группы $G$ тогда и только тогда, когда $V / G_{\mathfrak{g}}$ является (ङ-инъектором группы $G / G_{\mathfrak{g}}$;

(2) в любой группе $G$ существует единственный класс сопряжсенных §-инъекторов;

(3) §-инъекторы группы $G$ - это в точности все те §-максимальные подгруппы $G$, которые содержат $\mathfrak{F}$-радикал $G_{\mathfrak{F}}$.

Доказательство теоремы осуществляется аналогично доказательству теоремы 1 с учетом леммы 3 и утверждения 2 леммы 1.

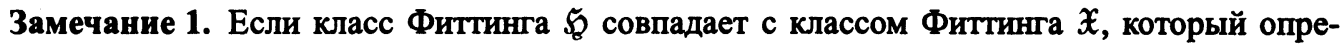
деляет класс $H R(f, R)$, то ввиду леммы IX.3.19 из [2] условие ङ-скованности для факторгрупшы $G / G_{\mathfrak{X}}$ можно опустить. В этом случае, учитывая лемму 3 , получаем, что в любой группе $G \in \mathfrak{S}^{\pi}$ существует единственный класс сопряженных $\mathfrak{X}$ которые в точности являются $\mathfrak{X}$ Б-максимальными подгруппами $G$, содержащими $\mathfrak{X}$ (ङ-радикал $G_{\mathfrak{X}}$.

\section{4. Следствия теорем 1 и 2}

Вначале приведем следствия из теоремы 1, которые получаются с помощью задания конкретных значений композиционной $H$-функщии $f$, определяющей конструкцию Гашюца $H R(f, R)$. 
Пусть классы $\mathfrak{X}, \mathfrak{V}$ и $\mathfrak{L}$ таковы, что $\mathfrak{X}=(1), \mathfrak{V}=\mathbb{E}$ и $\mathfrak{L}=\mathfrak{I}$. Определим композиционную $H$-функцию следующим образом:

$$
f(J)= \begin{cases}(1), & J \in \mathfrak{R} \cap \mathfrak{A}, \\ D_{0}(J), & J \in \mathfrak{L} \backslash \mathfrak{A} .\end{cases}
$$

Тогда ввиду того, что по леммам III.4.3 и X.13.12 из [7] каждая группа $\mathfrak{N}^{*}$-скована, для класса $H R(f, R)=\mathfrak{N}^{*}$ мы получаем следующее утверждение.

Следствие 1. В любой группе $G$ существуют $\mathfrak{S}_{\mathfrak{1}}^{*}$-инъекторы и любые два из них сопряжены в $G$.

В случае, когда $\mathfrak{S}=(1)$, из следствия 1 вытекает следующее утверждение, доказанное B [6].

Следствие 2. В любой группе $G$ существуют квазинильпотентные инъекторы, и любые два из них сопряжены в $G$.

Приведем теперь следствия из теоремы 1, которые получаются при конкретных значениях классов $\mathfrak{X}, \mathfrak{V}$ и $\mathfrak{L}$, определяющих $B L$-класс $\mathfrak{B}$.

Пусть $\mathfrak{X}=\mathfrak{E}, \mathfrak{V}=(1)$, причем $\mathfrak{Q}-$ класс простых групा, который содержит все группы простого порядка, тогда согласно 5.2 из [6] получаем следующее утверждение.

Следствие 3. В любой группе $G$ такой, что $G / G_{\mathfrak{j}}$ является $\mathfrak{\Re - с к о в а н н о и ̆ , ~ с у щ е с т в у ю т ~}$ $\mathfrak{S} \mathfrak{⿰ - и н ъ е к т о р ы ~ и ~ л ю б ы е ~ д в а ~ и з ~ н и х ~ с о п р я ж е н ы ~ в ~} G$.

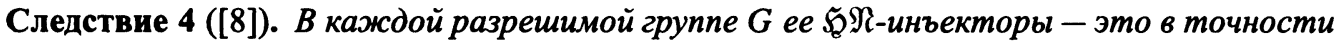
$\mathfrak{S} \Re$-максимальные подгруппы $G$, содержащие ее $\mathfrak{S}_{\mathfrak{N}}$-радикал.

Если же $\mathfrak{S}=(1)$, то из следствия 3 вытекает следующее утверждение.

Следствие 5 ([14]). Каждая $\mathfrak{\Re - с к о в а н н а я ~ г р у п п а ~ о б л а д а е т ~ е д и н с т в е н н ы м ~ к л а с с о м ~ с о - ~}$

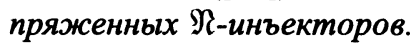

Следствие 6 ([9]). В каждой разрешимой группе $G$ ее нильпотентные инъекторы - это в точности все те из максимальных нильпотентных подгрупп $G$, которые содержат nодгруппу Фиттинга $F(G)$.

Наконец, в случае, когда $\mathfrak{X}=\mathfrak{N}, \mathfrak{Y}=(1)$, причем $\mathfrak{L}=\left\{\mathbf{Z}_{p}\right\}$, мы получаем для класса Фиттинга

$$
\mathfrak{A}=\left\{G \in \mathfrak{E} \mid O_{p}(G) \leqslant Z_{\infty}(G)\right\}
$$

следующие утверждения.

Следствие 7. В любой группе $G$ такой, что $G / G_{\mathfrak{g}}$ является $\mathfrak{\Re}$-скованной, существуют Ђু囚-инъекторы и любые два из них сопряжены в $G$.

Следствие 8 ([10]). В каждой разрешимой группе $G$ ее $\mathfrak{\Re}$-инъекторы-это в точности 凡-максимальные подгруппы $G$, содержащие $\mathfrak{\AA}$-радикал $G_{\mathfrak{R}}$.

Укажем теперь следствия, которые вытекают из теоремы 2 и описывают инъекторы для некоторых известных классов Фиттинга.

Пусть в дальнейшем $\pi=\operatorname{Char}(\mathfrak{L})$, где $\mathfrak{L}-$ подкласс класса $\mathfrak{I}$ всех конечных простых групп. Тогда в случае, когда $\mathfrak{X}=(1)$, учитывая замечание к теореме 2 , получаем следующее утверждение. 
Следствие 9 ([6]). В любой $\pi$-разрешимой группе $G$ существует единственный сопряженный класс $『$-инъекторов, каждый из которых в точности является 『-максимальной подгруппой группы $G$, содержащей ее $『$-радикал $G_{\S}$.

Пусть теперь классы $\mathfrak{X}, \mathfrak{V}$ и $\mathfrak{L}$, определяющие конструкцию Гашюца $H S(\psi, S)$, таковы, что $\mathfrak{X}=(1), \mathfrak{V}=\mathfrak{E}, \mathfrak{L}-$ класс всех неабелевых простых групп, и композиционная $H$-функция $\psi$ такова, что

$$
\psi(J)= \begin{cases}\mathfrak{E}, & J \in \mathfrak{Q} \cap \mathfrak{A}, \\ D_{0}(J), & J \in \mathfrak{Q} \backslash \mathfrak{A} .\end{cases}
$$

Тогда ввйду IX.2.9 (c) из [2] класс

$$
H S(\psi, S)=\mathfrak{\Re}=(G \in \mathbb{C} \mid[\operatorname{Soc}(G), \operatorname{Soc}(G)] \text { является прямым множителем } G)
$$

и справедливо следующее утверждение.

Следствие 10. В любой $\pi$-разрешимой группе $G$ такой, что $G / G_{\mathfrak{g}}$ является $\Re$-скованной, существует единственный класс сопряженных $\mathfrak{g} \Re$-инъекторов.

В случае, когда $\mathfrak{G}=(1)$ справедливо следующее утверждение.

Следствие 11. В любой $\pi$-разрешимой $\Re$-скованной группе $G$ существуют $\Re$-инъектоpы, и любые два из них сопряжены в $G$.

Теперь приведем следствия из теоремы 2, которые получаются при конкретных значениях классов $\mathfrak{X}, \mathfrak{V}$ и $\mathfrak{L}$, определяющих $B L$-класс $\mathfrak{~}$

Пусть $\mathfrak{X}=\mathfrak{E}, \mathfrak{V}=(1)$, причем $\mathfrak{L}=\left\{Z_{p}\right\}$, тогда $B L$-класс

$$
\mathfrak{S}=乃^{p}=\left(G \in \mathbb{E} \mid \operatorname{Soc}_{p}(G) \leqslant Z(G)\right)
$$

и из теоремы 2 вытекает следующее утверждение.

Следствие 12. $B$ р-разрешимой группе $G$ такой, что $G / G_{\mathfrak{\zeta}}$ является $\mathfrak{Z}^{p}$-скованной, существуют $5 \Omega^{p}$-инъекторы, и любые два из них сопряжены в $G$.

Специальным случаем следствия 12 в классе всех разрешимых групп является результат Локетта-Франца $[11,10]$.

Заметим, что теорема 2 в общем случае в классе (5 неверна. Действительно, если $\mathfrak{X}=\mathfrak{E}, \mathfrak{Y}=(1)$, причем $\mathfrak{L} \cap \mathfrak{A} \neq 0$, то согласно 4.6 из [6] найдется группа $G$, в которой существуют по крайней мере два класса сопряженных $C_{\mathfrak{L}}^{K}(\&,(1))$-инъекторов. 


\section{Список литературы}

1. Fischer B., Gaschutz W., Hartley B., Injektoren endlicher auflosbarer Gruppen. Math. Z. (1967) 102, №5, 337-339.

2. Doerk K., Hawkes T., Finite soluble groups. Walter de Gruyter, Berlin, 1992.

3. Шеметков Л. А., О подгруппах $\pi$-разрешимых групп. В кн.: Конечные группы. Наука и техника, Минск, 1975, с. 207-212.

4. Шеметков Л. А., Некоторые свойства инъекторов конечных групп. Изв. Гомельского гос. ун-та им. Ф. Скорины, Вопросы алгебры. (1999) 15, №1, 5-13.

5. Сементовский В. Г., $\Delta$-нильпотентные инъекторы конечных групп. Bonросы алгебры (1985) 1 , 72-86.

6. Blessenohl D., Laue H., Fittingklassen endlicher Gruppen in denen gewisse Haupfaktoren einfach sind. J. Algebra (1979) 56, 516-532.

7. Huppert B., Blackburn N., Finite groups. III. Springer, Berlin, 1982.

8. Hartley B., On Fisher's dualization of formation theory. Proc. London Math. Soc. (1969) 3, №2, 193-207.

9. Fischer B., Klassen konjugierter Untergruppen in endlichen auflosbaren Gruppen. Habilitationschrift, Univ. Frankfurt, 1966.

10. Frantz W., Spezielle Fittingklassen und ihre Injektoren. Diplomarbeit, Kiel, 1970.

11. Lokett P., On the theory of Fitting classes of finite soluble groups. Ph. D. Thesis, Univ. Warwick, 1971.

12. Шеметков Л. А., Формачии конечных групп. Наука, Москва, 1978.

13. Iranzo M. J., Perez Monasor F., $\mathfrak{F}$-constraint with respect to a Fitting class. Arch. Math. (1986) 46, 205-210.

14. Mann A., Injectors and normal subgroups of finite groups. Israel J. Math. (1971) 9, 554-558.

15. Laue P., Über nichtauflosbare normale Fittingklassen. J. Algebra (1977) 45, 274-283.

Статья поступила 03.04.2003. 